\section{THE CORDAT LAB}

\section{Eureka}

Eureka Team

Emmanuelle Cordat is an assistant professor in the Department of Physiology. A recent paper of hers was accepted by Biochemical Journal Feb 2010: "Band 3 Edmonton I, a novel mutant of the anion exchanger 1 causing spherocytosis and distal renal tubular acidosis". Below is a review from the lead author of this paper, Carmen Chu.

Distal renal tubular acidosis is a disease of the kidney that leads to formation of kidney stones, failure in urine acidification, failure to thrive and eventually renal failure. There is no treatment for this disease. Spherocytosis is a disease of the red blood cells, causing the affected individual to become anemic and to require regular blood transfusions until removal of the spleen eventually resolves the anemia problem. This novel mutant of the anion exchanger 1 (AE1) was characterized from a patient of the University of Alberta Stollery Children's Hospital. Studying the trafficking patterns of the mutant anion exchanger I in cell lines proteins revealed that the localization of the mutated protein was different from the wildtype, and that abnormal localization correlated to the disease phenotypes displayed by the patient and family members. Due to its abnormal localization in cells, this novel mutant is likely unable to perform normal function in the kidney and thus causes the disease in this patient. This investigation was led by Dr. Emmanuelle Cordat, and the Cordat lab are now working on other aspects of the anion exchanger I.

Currently, the lab is working on several aspects of the kidney anion exchanger I (kAEI). The restoration of intracellularly retained mutant kAEI trafficking to the plasma membrane is one area of interest. Indeed, re-establishment, even if partial, of normal trafficking may restore partial function of the protein and improve physiological conditions of affected individuals. Inspiration as to which small molecules to test first were drawn from those that restored trafficking of $\triangle F 508$ CFTR, vasopressin 2 receptor (V2R), and aquaporin 2 mutants. Results thus far have shown success in rescue for some mutants of kAEI, thus bringing potential opportunities for new therapies for these patients. The affect of these small molecules on the interaction of kAEI with chaperone proteins are also being studied.

Another area of research in the Cordat lab is looking at is the role of interacting proteins of AEI on trafficking. The mutant forms of AEI may be localized to the wrong place in cells because they are unable to interact with trafficking proteins or in contrast, because they are interacting too strongly with some others. Such interactions could explain the mistargeting of various mutant proteins. Studying these proteins' interactions will give clues as to how mistargeting of mutant proteins can be repaired.

Finally, previous studies have shown that the C-terminus of kAEI has important interacting partners that are crucial to proper targeting of kAEI. One such protein that we are studying is the adaptor protein 1A (AP-1A), which resides in the Golgi and endosomes. We suspect that the lack of interaction between AP-1A and kAE1 mutants could cause distal renal tubular acidosis in certain patients.

Stay tuned for more updates on the Cordat lab!! 